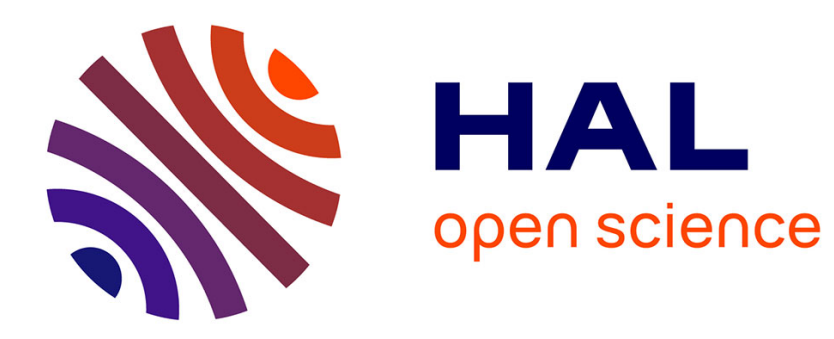

\title{
La signification des expressions égocentriques
}

Jérôme Dokic

\section{- To cite this version:}

Jérôme Dokic. La signification des expressions égocentriques. Joëlle Proust. Perception et intermodalité. Approches actuelles de la question de Molyneux, PUF, Paris, pp.103-124, 1997. ijn_00000270

\section{HAL Id: ijn_00000270 \\ https://hal.science/ijn_00000270}

Submitted on 4 Nov 2002

HAL is a multi-disciplinary open access archive for the deposit and dissemination of scientific research documents, whether they are published or not. The documents may come from teaching and research institutions in France or abroad, or from public or private research centers.
L'archive ouverte pluridisciplinaire HAL, est destinée au dépôt et à la diffusion de documents scientifiques de niveau recherche, publiés ou non, émanant des établissements d'enseignement et de recherche français ou étrangers, des laboratoires publics ou privés. 
[Paru in J. Proust (dir.), Perception et intermodalité: approches actuelles de la question de Molyneux, Paris: PUF, 1997.]

\title{
LA SIGNIFICATION DES EXPRESSIONS EGOCENTRIQUES*
}

\author{
Jérôme Dokic \\ (CREA; Université de Genève)
}

\begin{abstract}
0. Les expressions égocentriques sont des termes indexicaux typiquement utilisés pour spécifier une région du champ perceptif se trouvant dans une certaine relation au corps propre, considéré comme étant au centre du champ en question. "A gauche", "à droite", "en haut", "en bas", "devant", "derrière" et "ici" (qui désigne le centre de l'espace perceptif) forment ainsi une famille d'expressions égocentriques, qui compte également des expressions composées telles que "en haut à droite".

J'aimerais proposer ici, non pas une analyse définitive du fonctionnement de ces expressions, mais quelques remarques préliminaires au sujet de leur signification. La structure de l'article est la suivante. Je commence par rappeler certaines remarques de Gareth Evans sur la signification des expressions égocentriques. D'un mot, celle-ci est liée de manière privilégiée à l'action, et suppose l'existence d'un espace égocentrique (section 1). Toutefois, la notion d'espace égocentrique est problématique (section 2). On peut essayer de définir cette notion par référence à une conception absolutiste (vs. relationaliste) de la position dans le champ perceptif (section 3). Certains arguments en faveur de cette conception, inspirés de Wittgenstein, sont examinés avec un oeil critique (sections 4 et 5). Un autre argument est rejeté, et un type de lien important entre perception et action est mis en évidence (section 6). Dans la conclusion (section 7), je tire les conséquences pertinentes de la discussion pour l'évaluation de la théorie d'Evans présentée au commencement.
\end{abstract}

1. En guise de point de départ, considérons les deux thèses suivantes sur la signification des expressions égocentriques, proposées par Evans dans son livre The Varieties of Reference:

(i) "La signification de ces termes [égocentriques] est dérivée en partie de connexions complexes entre ceux-ci et les actions du sujet" (1982: 155).

(ii) L'information spatiale fournie par l'expérience perceptive "est spécifiable seulement dans un vocabulaire dont la signification des termes est dérivée en partie du fait qu'ils sont liés à des actions corporelles" (1982: 157; mes italiques. La citation d'Evans concerne l'expérience auditive, mais elle vise en définitive l'ensemble de l'expérience perceptive).

Selon la première thèse, il est impossible de spécifier la signification des expressions égocentriques, du moins lorsque leur utilisation est étroitement liée à l'expérience perceptive,

\footnotetext{
* J'ai profité de commentaires de Bill Brewer, John Campbell, Eros Corazza, Joëlle Proust, Gianfranco Soldati et Richard Vallée sur une version plus ancienne de cet article. Celui-ci a été présenté au séminaire de David Wiggins à Birkbeck College, Londres, à celui de Kevin Mulligan à Genève, et enfin lors d'une journée sur la philosophie de la perception organisée par Joëlle Proust à Paris en mars 1995. Je tiens à remercier les personnes mentionnées, ainsi que les participants à ces rencontres.
} 
sans mentionner à un moment ou à un autre le système d'actions possibles à la disposition du sujet. La différence entre la signification de "gauche" et celle de "droite", par exemple, ne peut être expliquée, en définitive, que par le biais de différentes attitudes comportementales à l'égard des régions de l'espace perceptif en question. Dans un contexte normal, je peux manifester ma saisie de la différence pertinente, par exemple en pointant mon doigt dans l'une ou l'autre direction.

Selon Evans, si les expressions égocentriques sont particulièrement appropriées à la description du contenu spatial de l'expérience, c'est que celle-ci est intrinsèquement orientée: elle possède un haut, un bas, une gauche, une droite, un arrière-plan et un avant-plan. L'espace de la perception est lui-même de nature égocentrique. C'est ici qu'intervient la seconde thèse, qui implique la notion technique d'information. Je rappelle que dans la terminologie d'Evans, l'information fournie par l'expérience perceptive est d'abord non conceptuelle, et que les jugements fondés sur la perception impliquent une conceptualisation de cette information (1982: 227). Ainsi, sur le plan préconceptuel déjà, l'information spatiale nous est donnée sous un mode égocentrique, et l'utilisation explicite des expressions égocentriques implique une conceptualisation de cette information. Je perçois un oiseau comme étant perché sur l'arbre en haut à droite, et cette expérience me permet de fonder un jugement égocentrique du type "L'oiseau s'est perché sur l'arbre en haut à droite". Suivant la thèse (ii), toute description non égocentrique du contenu spatial de l'expérience serait fondamentalement incomplète, puisqu'elle ne refléterait pas ce qui fait la spécificité du contenu en question.

Par ailleurs, la seconde thèse fournit chez Evans une sorte de fondement préconceptuel à la première thèse. En effet, les connexions entre perception et action existent déjà avant toute conceptualisation de l'information spatiale: "Une entrée perceptive [...] ne peut avoir une signification spatiale pour un organisme que dans la mesure où elle se place dans un réseau complexe de connexions entrées-sorties" (1982: 154). Or non seulement l'expérience consciente suppose déjà la mise en place d'un tel réseau, mais celui-ci est responsable de son orientation intrinsèque. Evans reprend à son compte l'affirmation de Charles Taylor selon laquelle "la structure d'orientation marque notre champ [perceptif] comme étant essentiellement celui d'un agent incarné" (1978-9: 154). On est également proche de la thèse de Merleau-Ponty selon laquelle "ce qui importe pour l'orientation du spectacle, ce n'est pas mon corps tel qu'il est en fait, comme chose dans l'espace objectif, mais mon corps comme système d'actions possibles" (1945: 289). Il n'est donc pas étonnant que les expressions que nous utilisons pour décrire le contenu spatial de l'expérience aient elles-mêmes un lien étroit avec les actions du sujet: en fait, selon Evans, il doit y avoir une "harmonie entre les pensées et le comportement qui résulte d'un état sensoriel donné" (1982: 159). Autrement dit, la thèse (i), selon laquelle la signification conceptuelle des expressions égocentriques ne peut être spécifiée que par le biais de capacités comportementales, est fondée sur la thèse analogue, qui concerne le plan préconceptuel, selon laquelle l'orientation de la perception découle de liens complexes entre des états sensoriels et des réponses comportementales.

2. On peut supposer que l'expérience perceptive, lorsqu'elle est véridique, nous présente un monde physique spatial, mais peut-on être plus explicite sur les traits de l'espace physique qui sont véritablement accessibles au sujet percevant? On peut dire que les traits en question concernent en tout cas des relations spatiales entre des positions potentiellement occupées par des éléments perçus. Un sous-ensemble appréciable des relations spatiales qui constituent l'espace entourant le sujet est normalement perceptible: je perçois une certaine distance visible entre la tasse et la cafetière, une autre distance visible entre la tasse et ma main, une autre relation de distance encore entre ma main et le reste de mon corps tel qu'ils 
sont perçus au travers du sens proprioceptif. ${ }^{1}$ Ce dernier point est compatible avec une théorie absolutiste de l'espace physique, selon laquelle la position dans cet espace n'est pas déterminée de manière univoque par un ensemble de rapports de position. Apparemment, Newton (1985: $\S 4)$ admettait même que seules des relations spatiales entre des positions sont accessibles au sujet percevant, les positions absolues dans l'espace physique n'étant pas perceptibles en tant que telles.

A ce stade, un problème potentiel se pose pour le défenseur de la thèse selon laquelle seul le vocabulaire égocentrique spécifie de manière adéquate l'information spatiale fournie par l'expérience - thèse (ii) de la section précédente. En effet, pour autant qu'elle concerne des relations spatiales physiques, cette information peut apparemment être décrite sans utiliser le vocabulaire égocentrique. Par exemple, je perçois une certaine relation spatiale que je décris de la façon suivante: "Cette tasse est à telle distance de cette cafetière", où les termes déictiques "cette tasse", "cette cafetière" et "telle distance" sont fondés sur la perception. Il n'est pas évident que ces termes déictiques (ici, il faut compter aussi des termes comme "cette position/région") soient de type égocentrique, puisqu'ils ne spécifient pas la position d'un objet en relation avec le corps propre du sujet. Si je décris la tasse comme étant à telle position et la cafetière à telle autre position, je ne peux rien en déduire sur la façon dont ces objets sont orientés par rapport au sujet. Il en va autrement si je décris la tasse comme étant à gauche et la cafetière à droite. ${ }^{2}$

Mais dans ce cas, on peut se demander en quoi l'espace de la perception est de nature intrinsèquement égocentrique. Certes, la position d'un objet dans cet espace dépend, entre autres, de relations qui impliquent le corps du sujet - perçu surtout au travers de la vue et du sens proprioceptif. Il ne paraît pas nécessaire, toutefois, d'identifier le corps en question comme mon corps. Je ne suis pas obligé de dire "Cette tasse de café est à telle distance visible de ma main", mais je peux m'en tenir à "Cette tasse de café est à telle distance visible de cette main". En ce qui concerne le contenu spatial de la perception, la première description n'ajoute rien à la seconde. Des remarques similaires valent pour la description "Cette main est à telle distance du reste de ce corps", où le terme "telle distance" est fondé cette fois sur le sens proprioceptif. Si l'espace de la perception est centré sur le corps, c'est dans un sens purement extensionnel: la scène perçue est telle que le corps du sujet occupe ce qui peut être considéré comme son centre géométrique. ${ }^{3}$

De toute évidence, le raisonnement qui précède repose sur la prémisse selon laquelle une description strictement relationnelle (c'est-à-dire, qui ne comporte pas d'expressions

\footnotetext{
${ }^{1}$ Je suppose dans ce texte que les relations spatiales entre objets perçus sont accessibles au sujet d'une expérience perceptive, même s'il s'avère qu'elles ne sont pas perceptibles exactement au même sens où la tasse et la cafetière le sont.

${ }^{2}$ Le point qui est en jeu ici est valable également pour les expressions égocentriques relationnelles. Je suppose dans cet article que les expressions égocentriques sont monadiques (comme lorsqu'on dit que dans mon champ visuel, l'oiseau est à droite), mais elles ont également un usage relationnel, comme dans "La tasse est à gauche de la cafetière". Or il y a une différence logique entre une relation du type " $x$ est à telle distance de $y "$ et une relation égocentrique du type " $x$ est à gauche de $y "$. La première relation est symétrique: par exemple, si la tasse est à une certaine distance visible de la cafetière, celle-ci est à la même distance visible de la tasse. (Je ne veux pas dire, évidemment, que toutes les relations spatiales non égocentriques soient symétriques; la relation " $x$ est dans $y$ ", par exemple, ne l'est pas.) En revanche, la relation " $x$ est à gauche de $y$ " n'est pas symétrique: si la tasse est à gauche de la cafetière (dans un contexte perceptif donné), la cafetière n'est pas à gauche de la tasse (dans le même contexte). Or il est clair qu'à partir seulement d'une description du type "La tasse est à telle distance de la cafetière", on ne peut pas savoir si la tasse est à gauche ou à droite de la cafetière par rapport au sujet (dans un contexte donné).

${ }^{3}$ Cf. John Campbell (1994: 10), qui insiste sur la nécessité d'introduire une notion psychologique d'espace égocentrique, par opposition à une notion géométrique, qui serait purement extensionnelle. Il me paraît clair que la théorie d'Evans vise la première notion.
} 
égocentriques) peut être considérée comme un compte rendu exhaustif des traits spatiaux de ce qui est perçu. Le défenseur de la thèse (ii) contestera sans doute cette prémisse, arguant que l'expression "ce qui est perçu", au moins en ce qui concerne le contenu spatial de l'expérience, manifeste une ambiguïté de type sens/référence: l'expérience fait bien référence à un ensemble de relations spatiales non égocentriques, mais elle présente cet ensemble sous un mode déterminé, à savoir le mode égocentrique. Or une description strictement relationnelle ne peut pas "capturer" un tel mode. Seule une description qui reprend le vocabulaire égocentrique est susceptible de le faire.

Cet argument en faveur de la thèse (ii) n'est valable que si l'on peut définir une notion substantielle de mode de présentation égocentrique (sur le plan préconceptuel). Dans le cas contraire, l'argument reste purement verbal. La question pertinente est de savoir ce qui vient s'ajouter à la perception des relations spatiales non égocentriques pour constituer le contenu spatial de l'expérience. La stratégie que je vais adopter pour essayer d'y répondre est la suivante. Dans ce qui suit, je me propose d'examiner une notion substantielle de mode de présentation égocentrique, qui me paraît implicite dans la plupart des discussions philosophiques sur cette question. Nous verrons qu'en réalité, la notion examinée est contestable. D'autres notions de mode de présentation égocentrique peuvent être envisagées, mais une conclusion plus simple s'impose naturellement: l'expérience spatiale ne provoque pas d'ambiguïté de type sens/référence dans l'expression "ce qui est perçu". Les relations spatiales sont directement accessibles au sujet percevant, c'est-à-dire qu'elles ne sont pas présentées sous quelque mode de présentation que ce soit. Il s'ensuit que la notion d'espace égocentrique, c'est-à-dire la notion d'un espace orienté sur le plan préconceptuel, devient superflue. On peut certes utiliser le vocabulaire égocentrique pour décrire le contenu spatial de l'expérience, mais rien ne nous y oblige: une description strictement relationnelle ne serait pas essentiellement ambiguë, et serait susceptible de représenter de manière univoque la structure spatiale de l'expérience.

3. Selon la notion visée de mode de présentation égocentrique, le même espace peut être présenté sous différents modes égocentriques. Autrement dit, on peut faire varier la position apparente d'un objet - son orientation - sans modifier aucune relation spatiale non égocentrique présentée dans l'espace perceptif. La notion en question suppose donc une thèse absolutiste du champ perceptif: ${ }^{4}$

(S) Dans le champ perceptif, la position d'un objet ne survient pas sur l'ensemble des rapports (non égocentriques) de position.

La notion de survenance est entendue ici au sens habituel selon lequel, si un fait A survient sur un ensemble de faits B, alors toute différence dans le fait A implique nécessairement quelque différence dans l'ensemble des faits $\mathrm{B}$. La négation de $\mathrm{S}$ est une thèse relationaliste du champ perceptif, c'est-à-dire une thèse de la survenance entre l'orientation du champ perceptif et sa structure relationnelle: toute différence apparente dans la position d'un objet perçu est nécessairement accompagnée de quelque différence concernant les relations spatiales qui constituent l'espace perçu.

Notons que $\mathrm{S}$ peut être acceptée par un philosophe qui rejette la notion de position absolue dans l'espace physique. C'est que les positions absolues dans le champ perceptif ne correspondent pas forcément à des positions absolues dans un espace physique qui dépasse les

\footnotetext{
${ }^{4}$ Pour une présentation succincte du débat entre les positions relationaliste et absolutiste sur l'espace de la perception, voir aussi Casati et Dokic (1994: ch. 7).
} 
limites de l'observation. Comme le disait Merleau-Ponty, l'orientation du champ perceptif n'est pas un reflet de celle du monde physique (même si l'expérience est véridique). Il ne s'ensuit pas que la position égocentrique d'un objet perçu soit un élément (systématiquement) illusoire de l'expérience; à nouveau, mieux vaut dire que l'orientation relève, non pas de ce qui est littéralement présenté dans l'expérience, mais du mode de présentation inhérent à celle-ci. Dans le même esprit, Evans écrit que l'information spatiale fournie par l'expérience "n'est pas une information qui concerne un type spécial d'espace, mais un type spécial d'information qui concerne l'espace" (1982: 157).

4. On vient d'introduire une notion possible de mode de présentation égocentrique, mais son intelligibilité dépend de la supériorité théorique de $\mathrm{S}$ par rapport à son antithèse relationaliste. On trouve dans les Remarques philosophiques ${ }^{5}$ de Wittgenstein une série d'arguments qui pourraient être repris dans le cadre d'une défense de l'absolutisme. Wittgenstein écrit par exemple, au sujet du champ visuel:

$[\mathrm{N}] \mathrm{e}$ pourrions-nous pas nous imaginer un espace visuel dans lequel on ne percevrait que certains rapports de positions, mais non pas une position absolue? [...] Je ne le crois pas. On ne pourrait pas par exemple percevoir une rotation de l'ensemble de l'image visuelle, ou plutôt elle ne serait pas pensable. Parlons de l'aiguille d'une montre se mouvant tout le long du cadran. (Je suppose que le cadran, comme c'est le cas dans beaucoup de grosses montres, ne porte que des points sans chiffres). Certes nous percevrions alors le mouvement d'un point à un autre - s'il ne se produit pas d'un coup - mais, l'aiguille une fois parvenue en un point, nous ne pourrions pas distinguer sa position de celle qu'elle avait au point précédent (1975: §206).

Comme nous le verrons plus en détail dans la section 6, il convient en réalité de distinguer deux aspects de l'argument de Wittgenstein. Le premier concerne notre capacité de percevoir des symétries, alors que le second aspect est lié à la possibilité d'effectuer des inversions perceptives détectables, et en particulier des rotations de l'"ensemble de l'image visuelle". ${ }^{6}$ Examinons pour l'instant ce dernier aspect.

Il est clair qu'une rotation de l'ensemble de l'image visuelle est possible, et même dans certains cas réelle. Les lunettes que Stratton (1897) a utilisées dans sa célèbre expérience, formées de lentilles biconvexes, inversaient précisément le haut et le bas, ainsi que la gauche et la droite. Comme on peut se l'imaginer aisément, il y a une différence phénoménologique remarquable entre l'image visuelle avant et après le port de ces lunettes (avant même que toute adaptation ait lieu). Or le fait intéressant est que cette inversion préserve l'ensemble des relations spatiales dans le champ visuel: le plafond reste à la même distance visible du plancher, même si l'orientation de ces éléments a changé: le plafond qui était en haut se retrouve en bas, et le plancher qui était en bas se retrouve en haut. On sait comment MerleauPonty s'est inspiré des travaux de Stratton pour démontrer qu'en matière de perception spatiale, "nous avons besoin d'un absolu dans le relatif", puisque "toutes les relations

\footnotetext{
${ }^{5}$ Notons que dans ce texte, Wittgenstein fait explicitement le lien entre la notion d'orientation et celle de position absolue (dans le champ visuel): "Dans l'espace visuel, il y a position absolue, d'où aussi mouvement absolu. [...] On peut dire également que l'espace visuel est un espace orienté, un espace dans lequel il y a un haut et un bas, une droite ou une gauche" (1975: §206).

${ }^{6}$ Je reprends ici la métaphore wittgensteinienne de l'image, que j'utiliserai plus tard dans l'expression "l'ensemble de l'image perceptive". Cette métaphore ne doit pas être prise au pied de la lettre, sinon elle constituerait une pétition de principe contre le point de vue relationaliste: une image photographique, par exemple, peut être orientée de différentes façons, alors que les relations spatiales entre ses éléments sont préservées.
} 
objectives du corps et de l'entourage sont conservées dans le nouveau spectacle", c'est-à-dire après avoir chaussé les lunettes inversantes (1945: 287 et 286).

La possibilité évidente d'une inversion de l'ensemble de l'image visuelle est-elle en contradiction avec la thèse relationaliste? A ce stade, il faut être précis: une telle inversion montre seulement que la position dans l'espace visuel ne survient pas sur l'ensemble des relations spatiales qui ont pour termes des positions visuelles. La même structure relationnelle de l'espace visuel peut avoir des orientations différentes. Toutefois, un défenseur de la thèse relationaliste pourrait arguer que ce fait n'implique pas encore $S$, qui concerne l'ensemble de l'image perceptive (c'est-à-dire le champ perceptif, et pas seulement le champ visuel). Or si l'inversion de Stratton laisse inchangées les relations spatiales qui ont pour termes des positions visuelles, elle bouleverse les relations spatiales entre les positions visuelles et des positions accessibles au travers d'autres modalités sensorielles. Avant l'inversion, le plafond visible entretenait une certaine relation de proximité avec ma tête sentie, relativement à la distance plus grande qu'il entretenait avec mes pieds sentis. Mais juste après avoir chaussé les lunettes, ces relations spatiales-là ne sont pas préservées: par exemple, le plafond visuel entretient maintenant une relation de proximité relative avec mes pieds tels que je les perçois au travers du sens proprioceptif. ${ }^{7}$

Il est raisonnable d'admettre que les relations spatiales dont les termes sont des éléments perçus au travers de différentes modalités sensorielles - appelons-les des relations spatiales synesthésiques - sont accessibles à l'expérience au même titre qu'une relation spatiale de distance entre deux objets visuels. La reconnaissance de la synesthésie spatiale est liée à une interprétation substantielle de l'unité des sens. Nos sens nous présentent un monde unifié non pas seulement parce qu'ils nous fournissent des informations sur ce qui est en fait le même monde, mais surtout parce que le champ perceptif est constitué de relations spatiales en quelque sorte "trans-modales", puisqu'elles ne dépendent pas du fait que leurs termes sont des éléments perçus au travers de la même modalité sensorielle. Certes, il reste à dégager les conditions dans lesquelles la synesthésie spatiale est possible, mais il suffit pour l'instant de noter l'existence de nombreux cas réels de synesthésie spatiale. Par exemple, je tiens un objet dans la paume de ma main. Je vois une moitié de l'objet et j'en touche l'autre. J'ai l'expérience synesthésique d'un seul objet dont je perçois directement et pleinement la forme générale, et non pas de deux parties discontinues du même objet.

Dans sa Nouvelle théorie de la vision, Berkeley affirme que "la position d'un objet quelconque n'est déterminée que par rapport aux objets du même sens" (1985: §111). Il s'ensuit que la notion de distance perceptible entre un objet de la vue et un objet du toucher est à proprement parler inintelligible. Mais l'affirmation de Berkeley dépend d'une théorie contestable de la distance visible, selon laquelle la distance entre deux objets de la vue, par exemple, dépend du nombre de minima visibilia qui les séparent. Indépendamment de la notion suspecte de "minimum visibile" (qui ne varie pas avec l'acuité visuelle du sujet), cette théorie s'applique difficilement à un espace visuel tridimensionnel: si je vois Pierre exactement devant moi, et loin derrière lui un arbre, vois-je plus de minima visibilia entre Pierre et l'arbre qu'entre deux objets de la vue placés à quelques centimètres l'un de l'autre tout près de moi? Par ailleurs, comme le fait observer Peacocke (1989), une perception normale de

\footnotetext{
${ }^{7}$ Comment est-il possible, dans ces conditions, de parler avec les psychologues d'un conflit entre les données propres à différentes modalités sensorielles? Le port de lunettes inversantes engendre effectivement une situation de conflit, au sens où le sujet constate la présence d'illusions locales: par exemple, il perçoit un fantôme de tête visible là où il ne sent que ses pieds. Théoriquement, il existe plusieurs façons de résoudre ce conflit principalement, en mettant entre parenthèses (par un processus qui s'apparente peut-être à celui de la réduction perceptive) un ensemble de données sensorielles, visuelles ou proprioceptives. La question de savoir comment le conflit est résolu en fait est du ressort de la psychologie expérimentale.
} 
la distance n'implique pas d'unités de mesure: je ne vois pas la distance entre deux objets de la vue comme faisant tant de pouces ou tant de centimètres. Pour la même raison, je dirais que je ne vois pas cette distance comme étant constituée de tant de minima visibilia: telle distance m'est directement accessible dans la perception. Ce dernier point rend problématique la notion même de minimum visibile, censée être d'ordre phénoménologique. Même si je ne vois pas directement telle distance en termes de centimètres, je peux la mesurer comme faisant, mettons, dix centimètres. Mais si je ne vois pas directement telle distance comme étant constituée d'un nombre défini de minima visibilia, quelle procédure pourrait me permettre de déterminer ce nombre? Aucune règle physique ne peut être utilisée pour compter le nombre de minima visibilia entre deux points de mon champ perceptif.

Intuitivement, notre expérience de la distance est amodale, c'est-à-dire qu'elle n'est pas interne à telle ou telle modalité sensorielle. Certes, elle est parfois grossière, et spécifiable seulement en termes de proximité relative (par exemple, je peux percevoir le plafond visible comme étant simplement plus proche de ma tête sentie que de mes pieds sentis), mais elle suffit normalement à ordonner spatialement les champs sensoriels les uns par rapport aux autres. ${ }^{8}$ Berkeley:

Notons que sur ce point précis, la position de Wittgenstein est assez proche de celle de

[L]a position dans l'"espace senti" (comme je l'appellerai) [il s'agit de l'espace proprioceptif] n'a rien à voir avec la position dans l'espace visuel, elles sont indépendantes l'une de l'autre et, si l'orientation dans l'espace visuel n'était pas absolue, on ne saurait d'aucune façon établir une corrélation entre elle et celle de l'espace senti (1975: §207)

D'un point de vue relationaliste, Wittgenstein renverse tout simplement l'ordre de l'explication. Il n'est pas nécessaire d'établir après coup une corrélation entre l'espace visuel et l'espace proprioceptif puisqu'une expérience normale présente déjà un espace unifié par des relations spatiales synesthésiques. Wittgenstein se fourvoie précisément en attribuant une orientation absolue à un champ visuel coupé de ses relations synesthésiques avec le reste du champ perceptif. Or selon la réponse relationaliste envisagée, aucun champ sensoriel ne possède d'orientation absolue; celle-ci survient toujours sur un système de relations spatiales dont certaines peuvent être synesthésiques.

5. On a de bonnes raisons de penser que les objections contre la thèse relationaliste qui se fondent sur la possibilité d'inverser l'image visuelle dans son ensemble peuvent être levées par référence au phénomène de la synesthésie spatiale. En reconnaissant l'existence de relations spatiales synesthésiques, on peut maintenir, semble-t-il, une thèse de la survenance entre la position d'un objet dans le champ visuel et l'ensemble des relations spatiales synesthésiques accessibles au sujet percevant. On pourra objecter, à ce stade, que nous n'avons fait que repousser le problème. Si des cas d'inversion de l'image visuelle dans son ensemble

\footnotetext{
${ }^{8}$ La distance n'est pas le seul exemple de relation spatiale synesthésique; la co-localisation en est un autre. Par exemple, je perçois normalement une relation de co-localisation entre ma tête visible et ma tête sentie. Notons par ailleurs un cas où les relations spatiales synesthésiques entre l'espace visuel et l'espace proprioceptif sont franchement grossières. Wittgenstein affirme que lorsque je regarde une figure très éloignée à travers une longuevue, il ne m'est pas possible de "comparer sa position à celle de mon corps" (1975: §206). Mais cette affirmation est ambiguë: elle est vraie si elle signifie que je ne peux pas établir la distance exacte entre la figure et mon corps; elle est fausse si elle veut dire que je ne perçois pas de relation de proximité relative entre le haut visuel de la figure et le haut proprioceptif de mon corps.
} 
sont envisageables, pourquoi ne pas imaginer des cas d'inversion de l'ensemble de l'image perceptive? Or si ce dernier type d'inversion est possible, certaines différences d'orientation ne seront pas liées à des différences relationnelles, et le défenseur de la thèse absolutiste $S$ sera en meilleure posture que son adversaire relationaliste.

Une première remarque est qu'en supposant la possibilité d'une inversion de l'image perceptive dans son ensemble, la thèse absolutiste paraît nettement moins intuitive qu'au commencement. Si une inversion de l'espace visuel, ou de celui de l'audition (au moyen d'une sorte de "pseudophone" qui inverserait le haut et le bas, ainsi que la droite et la gauche, du champ auditif), est facilement envisageable, il est beaucoup plus difficile de se représenter une inversion (ou une rotation) globale du champ perceptif. Mais alors, comment décider si une telle inversion ferait une différence phénoménologique, à supposer que l'ensemble des relations spatiales du champ soit préservé?

Essayons de répondre à cette question par le biais du Gedankenexperiment suivant. Supposons une expérience perceptive normale dans laquelle on peut distinguer différents champs ou espaces sensoriels:
a. champ visuel
b. champ auditif
c. expérience de la verticalité (dont le fondement est dans l'oreille interne) ${ }^{9}$
d. espace proprioceptif (schéma corporel)

Pour simplifier, nous supposerons des espaces considérés à un instant donné et qui comportent au plus deux dimensions, mais les résultats obtenus ne dépendront pas, je l'espère, de ces simplifications.

Tous ces champs possèdent un axe haut/bas (c'est pourquoi on laisse de côté l'odorat et le goût), même si comme le font observer les psychologues, la discrimination auditive entre le haut et le bas est très mauvaise par rapport à celle entre la gauche et la droite. Tous possèdent également un axe gauche/droite, sauf peut-être l'expérience de la verticalité. L'expérience tactile est laissée de côté tant son orientation est liée à celle du champ proprioceptif. ${ }^{10}$ Supposons à présent l'existence d'un procédé, approprié à chaque champ, d'inversion de celuici selon tous ses axes égocentriques:

a'. inversion du champ visuel: ce qui était perçu en haut (respectivement, à droite) dans le champ visuel est à présent perçu en bas (respectivement, à gauche) dans le champ visuel.

b'. inversion du champ auditif: les sons entendus en haut (respectivement, à droite) dans l'espace auditif sont à présent entendus en bas (respectivement, à gauche) dans cet espace.

c'. inversion de l'expérience de la verticalité: les données visuelles, auditives et proprioceptives sont maintenues en place, mais la force de la gravitation est sentie comme étant soudainement renversée.

Quelques remarques s'imposent au sujet de c'. En premier lieu, la formulation de l'inversion de l'expérience de la verticalité est assez différente des formulations a' et b' parce

\footnotetext{
${ }^{9}$ Ce type d'expérience est souvent négligé dans les analyses philosophiques de la perception, mais il est bien réel. Il permet entre autres au sujet de garder son équilibre dans diverses situations, en étant sensible (sur un plan préconceptuel) à la direction de la force de gravitation.

${ }^{10}$ et à l'action. Les sensations tactiles telles que la rugosité ou la dureté d'une surface ne sont accessibles qu'à un agent capable de déplacer le membre qui touche, ou d'exercer une pression sur cette surface.
} 
qu'il est probable que l'orientation de l'axe haut/bas telle que nous la déterminons au moyen des termes ordinaires "haut" et "bas" soit (normalement) définie par référence à cette expérience. Autrement dit, on ne peut pas décrire immédiatement (c'est-à-dire avant toute adaptation éventuelle) le renversement apparent de la force de gravitation en disant "Maintenant, la gravitation entraine les choses vers le haut". ${ }^{11}$ Deuxièmement, on ne doit pas prendre en considération ici les effets collatéraux d'une inversion de type c': si la force de gravitation était sentie comme étant soudainement renversée, on pourrait s'attendre à ce que les objets auparavant perçus comme étant "sur" la table, par exemple, "tombent" en direction de la force en question. On peut faire abstraction des effets collatéraux de ce genre en supposant un champ perceptif constitué seulement de formes colorées "abstraites", c'est-à-dire sans les implications causales caractéristiques des objets matériels.

d'. inversion du champ proprioceptif: alors que les données visuelles et auditives restent les mêmes, et que l'expérience de la verticalité ne change pas, la tête qui était sentie en haut est maintenant sentie en bas dans le champ proprioceptif, et vice-versa. De même, le bras qui était senti à gauche et maintenant senti à droite, et vice-versa.

La situation décrite sous d' est un peu curieuse: on aurait l'impression d'avoir la tête en bas sans avoir l'impression que le sang monte à la tête, c'est-à-dire sans la lourdeur caractéristique que l'on ressent normalement la tête en bas. Cela montre que dans une situation normale, les données proprioceptives sont sans doute modifiées par notre expérience de la verticalité. Les champs c et d sont donc étroitement liés entre eux. Qu'il faille néanmoins les distinguer paraît inévitable si l'on considère l'expérience proprioceptive d'un cosmonaute en apesanteur. En bref, si l'inversion de c ne peut se concevoir sans l'inversion de d ou vice-versa, on peut considérer ici un seul mécanisme d'inversion. L'argument qui suit ne dépend pas, je pense, de ce point. Nous nous situons en effet sur le plan d'une expérience de pensée. De manière générale, nous supposons que chaque inversion peut être effectuée indépendamment des autres, mais les faits indiquent que cette supposition est inexacte. Nos sens spatiaux ne fonctionnent pas indépendamment les uns des autres. Un dérangement spatial dans une modalité sensorielle, par exemple la vue, entrâne immédiatement, même si ce n'est parfois que de façon temporaire, un dérangement spatial dans une autre modalité sensorielle, par exemple l'ouïe. ${ }^{12}$

Supposons à présent qu'un sujet donné opère avec tous les procédés d'inversion en même temps. La question est de savoir si cette opération entraîne ou non une différence phénoménologique immédiate. Après réflexion, il semble que la réponse soit négative: l'image perceptive elle-même est invariante (d'un point de vue phénoménologique) sous l'opération envisagée. Même si chaque procédé utilisé isolément entraîne l'inversion d'un champ sensoriel relativement aux autres, tous les procédés utilisés simultanément ne modifient pas la position apparente dans le champ perceptif, précisément parce qu'ils préservent l'ensemble des relations spatiales synesthésiques entre les champs et donc leur orientation relative.

Considérons l'objection suivante contre cette réponse. Nous ne devons pas oublier que l'apparence perceptive est définie, en partie, par des liens préconceptuels complexes entre des entrées sensorielles et des sorties motrices. Or l'opération envisagée bouleverse de manière radicale les connexions entre l'expérience et le comportement réel du sujet. Pourquoi ce bouleversement ne serait-il pas lié, justement, à une différence phénoménologique

\footnotetext{
${ }^{11}$ En termes kripkéens, nous pouvons aussi dire que nous fixons les axes des différents champs sensoriels au moyen du vocabulaire égocentrique ordinaire, mais qu'une fois les axes fixés et les inversions effectuées, nous n'avons plus de garantie que le vocabulaire s'applique correctement.

${ }^{12}$ Sur le cas de la vue et de l'ouïe, cf. par exemple Freedman et Rekosh (1968).
} 
indépendante de la structure relationnelle de l'expérience? En fait, le comportement réel n'est révélateur du contenu apparent de l'expérience que s'il est contrôlé ou guidé par la perception. Si le sujet n'utilise ne serait-ce qu'un seul dispositif inversant, par exemple les lunettes de Stratton, il aura de sérieux problèmes, au moins dans un premier temps, à exercer des mouvements contrôlés par la perception visuelle. Il s'ensuit que son comportement maladroit ne peut pas être considéré comme la manifestation normale du contenu spatial de l'expérience. (Par ailleurs, nous ne pouvons rien prédire sur les possibilités d'adaptation des mécanismes perceptifs, c'est-à-dire sur l'éventualité que les connexions sensori-motrices se réharmonisent après un port prolongé et simultané des différents dispositifs d'inversion.)

En résumé, le défenseur de l'hypothèse relationaliste peut faire appel à l'expérience de pensée qui vient d'être envisagée pour tenter de démontrer que toute différence de position apparente dans le champ perceptif est nécessairement liée à quelque différence dans les relations spatiales qui constituent ce champ. La notion d'une inversion globale du champ perceptif qui soit néanmoins détectable d'un point de vue phénoménologique est incohérente. Notons que nous avons essayé d'envisager une inversion de deux axes du champ perceptif, à savoir les axes haut/bas et gauche/droite, mais la conclusion de l'expérience n'est pas différente si nous nous en tenons à un seul axe, par exemple l'axe gauche/droite. ${ }^{13}$ D'un point de vue géométrique, il est parfaitement possible d'inverser un espace tridimensionnel selon un axe donné sans toucher aux relations spatiales qui constituent cet espace. Le résultat de l'inversion serait comme une image-miroir de celui-ci. Mais notre expérience de pensée semble indiquer qu'une inversion de l'axe gauche/droite de l'ensemble de l'image perceptive ne changerait en rien la position apparente des objets perçus, dans la mesure où l'inversion préserverait toutes les relations spatiales accessibles au sujet dans son expérience.

6. Toutefois, comme je l'ai indiqué, l'argument de Wittgenstein en faveur de la thèse absolutiste comporte un autre aspect. Peut-être n'avons-nous aucune peine à imaginer un champ perceptif exactement symétrique selon un axe donné; la question est alors de savoir si le relationaliste est en mesure d'admettre cette possibilité. ${ }^{14}$ Dans un champ symétrique, tout élément se trouvant d'un côté de l'axe de symétrie correspond à un élément similaire se trouvant de l'autre côté. Mais si la position d'un élément du champ nous est donnée seulement par le type de relations spatiales qu'il entretient avec le reste des éléments du champ, comment pouvons-nous distinguer les deux côtés de l'axe de symétrie? C'est à ce problème que Wittgenstein fait allusion dans son exemple du cadran sans chiffres. L'exemple concerne le champ visuel, mais on peut en étendre l'application à l'ensemble de l'image perceptive: supposons un sujet doté d'un corps parfaitement symétrique selon l'axe gauche/droite, occupé à contempler le cadran en question, sans que rien ne vienne briser cette situation symétrique dans le reste du champ perceptif. Comment un tel sujet pourrait-il distinguer la position de l'aiguille à 9 heures de sa position à 3 heures?

Dans son argument, Wittgenstein semble partir du présupposé que les relations spatiales accessibles au sujet percevant sont identifiées indépendamment des termes de ces relations, c'est-à-dire des éléments ou des parties d'éléments sensibles localisés dans le champ

\footnotetext{
${ }^{13}$ Les lunettes monoculaires de Stratton inversaient le champ visuel selon les axes haut/bas et gauche/droite, mais pas selon les axes devant/derrière. Plus tard, des appareils binoculaires furent inventés qui inversaient les trois axes du champ visuel; cf. Ewert (1930). La seule inversion visuelle selon l'axe gauche/droite (obtenue à l'aide de lunettes à prismes) a également été étudiée en détail.

${ }^{14}$ En fait, la possibilité d'un champ perceptif exactement symétrique est hautement théorique; notre champ perceptif réel n'est presque jamais symétrique, en partie à cause de l'asymétrie caractéristique du champ gravitationnel. Il vaut toutefois la peine d'envisager cette possibilité pour répondre aux objections de Wittgenstein et préciser la position relationaliste.
} 
perceptif. Mais ce présupposé ne va pas de soi, même d'un point de vue phénoménologique: ce que le sujet voit immédiatement, ce ne sont pas (ou pas seulement) des entités universelles susceptibles d'être exemplifiées en différentes situations perceptives, mais des rapports concrets entre des objets numériquement déterminés. En fait, le relationaliste ferait mieux de rejeter le présupposé en question. Dans un contexte normal, la perception de la position de l'aiguille à 9 heures et à 3 heures est liée en partie à la perception de relations spatiales concrètes - par exemple, entre l'aiguille et certaines parties du cadran, non détachées mais numériquement déterminées, et dont le sujet peut garder la trace à travers le temps. Avant le mouvement, le point de départ de l'aiguille sur le cadran entretient une relation de proximité (voire de co-localisation) avec la pointe de l'aiguille; après le mouvement, le même point entretient avec celle-ci une relation déterminée d'éloignement. Cette description est parfaitement compatible avec la thèse maîtresse du relationaliste, selon laquelle la position dans le champ perceptif survient sur l'ensemble des relations spatiales accessibles au sujet percevant; en l'occurrence, le mouvement de l'aiguille entraîne nécessairement un changement dans le réseau des relations spatiales dans lequel elle se place.

Pour clarifier ce dernier point, considérons l'analogie suivante, qui concerne non pas le plan de la perception, mais celui de la pensée conceptuelle. Strawson admet explicitement la possibilité théorique d'un redoublement sur une vaste échelle des choses et des événements localisés dans un secteur déterminé de l'univers (cf. 1973: 21). Mais cette possibilité montre qu'une conception purement générale des relations spatiales n'est jamais suffisante pour identifier dans la pensée les objets qui constituent les termes de ces relations. Au fondement de notre conception ordinaire de l'espace, nous devons utiliser directement des expressions indexicales telles que "ceci" ou "cela", qui désignent des objets numériquement déterminés. L'utilisation de ces expressions fixe le cadre de référence et élimine ainsi l'ambiguïté spatiale. Toutefois, on peut retenir la solution de Strawson sans pour autant revenir au point de vue absolutiste selon lequel la position des objets matériels est une fonction de leur rapport (empiriquement non détectable) à un espace absolu. Sur le plan de la pensée conceptuelle aussi, il paraît possible de combiner un point de vue relationaliste avec la thèse selon laquelle nous n'identifions pas la position spatiale en utilisant seulement des notions universelles de relations spatiales.

Cette analogie nous permet de poser un autre problème, qui est de savoir ce qui joue le rôle, dans le cas de la perception, de l'utilisation des expressions démonstratives sur le plan du langage et de la pensée. Selon une hypothèse plausible, l'identification des positions dans l'espace de la perception est liée aux attitudes comportementales du sujet. Ce qui contribue à fonder notre faculté de différencier par la perception les régions de l'espace, c'est la capacité pratique qui nous permet entre autres de montrer du doigt une région-cible déterminée. Cette hypothèse est acceptable d'un point de vue relationaliste. Le fait que nous pouvons viser du regard une région quelconque du champ perceptif sans avoir à identifier au préalable la distance qui nous sépare de cette région n'empêche pas celle-ci d'être déterminée de manière strictement relationnelle. Cela reste vrai même si la région en question n'est délimitée par aucune figure. On ne peut donc pas accepter cet autre argument de Wittgenstein en faveur d'un espace perceptif absolu:

Que cela ait un sens de dire: "Cette partie d'une tache rouge est rouge" (quand cette partie n'est pas délimitée par une limite visible), cela dépend de ceci: qu'il y ait un lieu absolu. En effet, s'il peut être question dans l'espace visuel d'un lieu absolu, alors je peux également assigner une couleur à ce lieu absolu si son environnement est de la même couleur (1975: §206). 
Je peux identifier une partie relativement bien délimitée d'une tache uniformément rouge dans mon champ visuel par exemple en effectuant des mouvements oculaires qui "impriment" une forme à la partie en question. Une fois identifiée, pourtant, la position de cette partie dans l'espace de la perception est déterminée de manière univoque par l'ensemble des relations spatiales entre celle-ci et les autres positions du champ perceptif. ${ }^{15}$

Sur le plan de la pensée conceptuelle, Strawson a bien mis en évidence la dépendance mutuelle qui existe entre notre conception relationnelle des positions et notre conception indexicale d'objets numériquement déterminés. Pour répondre aux objections de Wittgenstein qui concernent nos facultés perceptives, il suffit de faire valoir que la perception des relations spatiales dépend de la capacité de viser par le comportement un objet (ou une partie d'objet) numériquement déterminé. (On peut certes viser du regard une région vide du champ perceptif, mais cet exploit dépend normalement de notre capacité de percevoir quelque relation entre cette région et d'autres positions occupées du champ perceptif - ne serait-ce que celle de notre corps propre.) On peut laisser ouverte, par contre, la question inverse de savoir dans quelle mesure la capacité de viser par le comportement un élément déterminé dépend de la perception consciente d'un ensemble de relations spatiales. En fait, selon des travaux expérimentaux récents, certains patients sont capables d'un comportement orienté vers une partie de leur corps sans avoir d'expérience consciente - qu'elle soit visuelle ou proprioceptive - de la partie en question. ${ }^{16}$

En dissociant la question de savoir comment les éléments sensibles sont localisés dans le champ perceptif, et celle de savoir comment nous identifions leur position, nous pouvons rendre compte d'autres contre-exemples apparents à la thèse relationaliste. Soit un objet isolé se déplaçant dans le champ visuel. (Ce peut être l'aiguille de Wittgenstein, mais cette fois sans cadran.) D'un point de vue relationaliste, tout déplacement apparent devrait être accompagné d'un changement dans les relations spatiales accessibles au sujet percevant. Or la mention des relations spatiales synesthésiques entre l'objet et le corps propre n'est pas suffisante, car sujet et objet peuvent se déplacer ensemble, et ainsi préserver leur distance et orientation relatives. Même dans ce dernier cas, toutefois, il nous est possible en principe de voir l'objet se déplacer (en même temps que nous). Comment rendre compte de ce fait en termes strictement relationnels?

La réponse est à chercher dans une structure essentielle du champ perceptif, à savoir la structure figure/fond. Nous voyons l'objet se déplacer en partie ${ }^{17}$ à cause du fait qu'il modifie sa relation spatiale avec l'arrière-plan (le fond indéterminé du champ perceptif, qui peut aussi contenir le corps propre du sujet), et plus précisément avec des parties non détachées de l'arrière-plan. Ces parties non détachées sont des éléments possibles de l'expérience en vertu du fait que le sujet possède la capacité (non nécessairement actualisée) de les viser par son comportement. De manière générale, le déplacement d'un objet dans le champ perceptif constitue une transformation locale de ce champ, contrairement aux transformations

\footnotetext{
${ }^{15}$ Ce qui vaut pour des éléments objectifs du champ perceptif vaut pour le corps propre. Nous avons la capacité d'identifier immédiatement un élément du champ comme étant notre corps propre, mais cette capacité ne devrait pas être décrite en termes d'"auto-localisation" (comme le fait Brewer (1992) dans un article par ailleurs admirable), comme si elle supposait un "ici" absolu dans le champ perceptif, duquel dépendraient toutes les autres positions de ce champ. Il vaut mieux parler d'une capacité d'"auto-identification". La position de mon corps propre, comme celle de tout autre élément du champ perceptif, survient sur le réseau des relations spatiales qui constituent le champ perceptif.

${ }^{16}$ Cf. par exemple Rossetti, Rode et Boisson (1995). Il reste que dans les cas examinés, les informations tactiles et proprioceptives sont utilisées "implicitement" (selon le vocabulaire cognitif en vigueur), ce qui laisse ouverte la possibilité qu'elles soient à leur base strictement relationnelles.

17 Je dis "en partie" parce qu'il est probable que la perception du mouvement soit également liée au fait que la cause du mouvement est localisée dans l'objet lui-même (au moins dans les cas typiques).
} 
envisagées dans la section précédente, qui concernaient l'ensemble de l'image perceptive. Cela explique que même d'un point de vue relationaliste, une transformation locale peut provoquer une différence phénoménologique. Les mêmes remarques valent pour d'autres transformations locales dans le champ perceptif, comme la rotation d'une figure de $180^{\circ}$, ou la transformation de celle-ci en une réplique énantiomorphe. ${ }^{18}$

7. Je ne prétends nullement avoir considéré tous les aspects de la controverse au sujet de $\mathrm{S}$ et de son antithèse relationaliste. Mon propos consiste plutôt à signaler en quoi cette controverse est pertinente pour déterminer correctement la signification des expressions égocentriques, et en particulier établir leur fondement préconceptuel (dans l'expérience). Tout d'abord, si la conception relationaliste du champ perceptif est correcte, une voie importante vers une définition substantielle d'un mode de présentation égocentrique, et partant d'un espace égocentrique, est bloquée. Il n'y a ni haut, ni bas, ni gauche, ni droite, ni avant-plan, ni arrière-plan absolus dans le champ perceptif.

Une définition adéquate d'un mode de présentation égocentrique doit donc s'accommoder de la conception relationaliste. Il s'agirait d'une notion inhabituelle de mode de présentation, selon laquelle le même espace ne pourrait pas être présenté sous deux modes différents (toute différence de mode de présentation impliquerait une différence relative à ce qui est présenté). Mais il n'est peut-être pas nécessaire de s'engager dans une définition de ce genre, dans la mesure où une autre option, plus simple, consiste à renoncer carrément à la thèse (ii) - l'idée selon laquelle l'espace préconceptuel de la perception est égocentrique.

Que veut-on dire en effet lorsqu'on parle d'un haut ou d'un bas, d'une gauche ou d'une droite, d'un avant-plan ou d'un arrière-plan relatifs dans le champ perceptif? Ce n'est pas que l'espace de la perception soit intrinsèquement orienté (avant toute conceptualisation); c'est plutôt que nous nous y orientons en y fixant non seulement des axes, mais aussi des directions. Or il existe a priori plusieurs façons de fixer des directions dans le champ perceptif. Par exemple, on peut fixer la direction haut/bas en termes qualitatifs, par référence à notre expérience de la verticalité (dans un contexte terrien ordinaire). On peut fixer la direction devant/derrière en exploitant des traits asymétriques caractéristiques du corps humain. Enfin, on peut fixer la direction gauche/droite en utilisant directement des expressions déictiques: une fois l'axe pertinent déterminé par référence au corps humain, la direction gauche/droite de cet axe (par opposition à la direction droite/gauche du même axe) peut être définie comme "la direction qui va de cette position-ci à cette position-là", où les termes déictiques "cette position-ci" et "cette position-là" désignent des points distincts sur l'axe en question. Après que les directions appropriées ont été fixées d'une manière ou d'une autre, on peut utiliser des expressions égocentriques monadiques pour spécifier une région se trouvant dans une certaine relation avec d'autres points de l'axe pertinent - qui passe typiquement par le corps du sujet. ${ }^{19}$

On voit donc qu'il n'y a rien de "spécial" dans l'information spatiale fournie par la perception, qui est de nature strictement relationnelle. Les directions ne sont pas données

\footnotetext{
${ }^{18}$ Deux figures sont des répliques énantiomorphes lorsqu'aucun déplacement local et rigide ne permet de les superposer exactement, bien qu'elles aient les mêmes propriétés relationnelles internes. Par exemple, la figure bidimensionnelle "F" et son image dans le miroir sont des répliques énantiomorphes (dans le plan). Ou encore, si on inverse l'axe gauche/droite du champ visuel, on peut transformer une figure visible asymétrique en sa réplique énantiomorphe. Sur la philosophie des figures énantiomorphes, cf. van Cleve et Frederick (1991).

${ }^{19}$ Il est certain qu'une analyse complète de la signification des expressions égocentriques doit mentionner d'autres usages possibles: comme dans la relation " $x$ est à gauche de $y$ par rapport à $z$ ", ou dans la relation comparative " $x$ est plus à gauche que $y$ ", ou encore dans la description superlative " $x$ est tout à droite". Pour une description détaillée des différentes règles d'usage qui gouvernent la signification ordinaire des termes égocentriques, cf. Vandeloise (1986).
} 
telles quelles dans la perception, mais c'est nous qui les fixons de différentes manières selon les cas. Certes, l'existence de liens préconceptuels complexes entre les entrées sensorielles et les sorties motrices peut être reconnue, mais elle ne crée pas d'ambiguïté particulière de type sens/référence dans l'expression "ce qui est perçu" (du moins en ce qui concerne la dimension spatiale de l'expérience). Par suite, le contenu spatial de l'expérience peut être entièrement spécifié sans utiliser directement d'expressions égocentriques du type "en haut à gauche", bien que selon toute évidence, il ne puisse être décrit sans utiliser d'expressions déictiques du type "cet objet", "cette région/position", "telle distance", etc.

On dispose à présent d'une meilleure compréhension de la thèse (i) avancée par Evans, selon laquelle la signification des expressions égocentriques tire son origine en partie de liens complexes avec l'action. Comme nous l'avons vu dans la section 6, il est probable que l'identification des positions ou des régions de l'espace perceptif soit fondée sur des attitudes comportementales déterminées. Or sur le plan préconceptuel, la pensée dont l'expression comporte des termes déictiques du type "cet objet" ou "cette position" dépend sans doute d'un acte d'attention fondé sur la perception et dirigé vers une position déterminée dans l'espace. Dans un contexte de communication, cet acte peut être accompagné d'un geste indicateur explicite (voire même remplacé par celui-ci) - cf. Kaplan (1989). Toutefois, même si ces termes déictiques ont un rôle sémantique très proche de celui des termes égocentriques, ils ne sont pas égocentriques stricto sensu. En effet, si leur utilisation véhicule de l'information sur le type de rapport spatial qui existe entre le sujet et la position visée, cette information n'est pas exactement la même que celle qui est véhiculée par l'utilisation d'expressions égocentriques. Lorsque je spécifie une région du champ perceptif comme étant à gauche, je localise cette région sur un axe égocentrique déterminé, et donc par rapport à d'autres positions possibles sur cet axe - entre autres, toutes les positions qui sont plus à droite. Considérons à présent une situation dans laquelle je spécifie une région de mon champ perceptif simplement comme "cette région", en portant mon attention sur celle-ci. Peut-être mon acte d'attention fixe-t-il un axe déterminé dans mon champ perceptif, à savoir celui qui passe par le front et la région visée. Mais aucune orientation de cet axe n'est présupposée par l'utilisation du terme "cette région". Comme on vient de le voir, une orientation égocentrique (c'est-à-dire des directions sur un axe) ne peut être fixée que par référence à une relation d'ordre entre des positions.

Il existe peut-être une autre différence entre les expressions égocentriques du type "en haut à gauche" et les termes déictiques comme "cette position". Certains philosophes (dont Evans) ont fait observer que l'utilisation des termes déictiques dans la pensée doit être fondée sur la perception immédiate, et donc sur la réception effective d'information perceptive. En revanche, il est apparemment possible de former une pensée égocentrique les yeux fermés, ou sur quelque chose qui n'est pas actuellement dans le champ perceptif: "Je me demande s'il se cache maintenant vers la gauche ou vers la droite". Les expressions égocentriques n'ont pas directement de force déictique puisqu'elles peuvent être utilisées sans monstration explicite, et leur emploi ne repose pas sur la réception effective d'information perceptive. L'explication de cette différence, si elle existe, peut être la suivante. A nouveau, pour comprendre l'utilisation présente d'expressions égocentriques, il faut remonter à une situation originaire dans laquelle le sujet a fixé de manière appropriée un certain nombre de directions dans son champ perceptif. Mais une fois les directions fixées, il semble que le sujet qui garde la trace de sa position dans l'environnement soit capable de former des pensées sur des régions de l'espace qui l'entoure même s'il n'en reçoit aucune information sur le moment.

On voit donc, pour résumer, que ce sont au mieux les expressions déictiques non égocentriques telles que "cet objet" ou "cette position" qui ont un commerce privilégié avec l'action. Les expressions égocentriques ne font qu'exploiter ce commerce antécédent. Leur 
utilisation n'a pas de rapport spécifique à l'action, qui s'ajouterait à celui qui concerne l'utilisation des expressions non égocentriques.

\section{BIBLIOGRAPHIE}

Berkeley, G., Oeuvres I, éd. par G. Brykman, Paris: PUF, 1985.

Brewer, B., "Agency and Self-Location", in Mind 101, 1992.

Campbell, J., Past, Space, and Self, Cambridge (Mass.): MIT Press, 1994.

Casati, R. et Dokic, J., La philosophie du son, Nîmes: Chambon, 1994.

Evans, G., The Varieties of Reference, éd. par J. McDowell, Oxford: Clarendon Press, 1982.

Ewert, P.H., "The effect of inverted retinal stimulation upon spatially coordinated behavior", in Genetic Psychology Monographs 7, 1930.

Freedman, S. et Rekosh, J., "The functional integrity of spatial behavior", in The neuropsychology of spatially oriented behavior, éd. par S. Freedman, Homewood (Illinois): Dorsey Press, 1968.

Kaplan, D., "Afterthoughts", in J. Almog, J. Perry et H. Wettstein (éds), Themes from Kaplan, Oxford: OUP, 1989.

Merleau-Ponty, M., La phénoménologie de la perception, Paris: Gallimard, 1945.

Newton, I., Les principes mathématiques de la philosophie naturelle, trad. franç. M.-F. Biarnais, Paris: Ch. Bourgois, 1985.

Peacocke, C., "Perceptual Content", in Themes from Kaplan, éd. par J. Almog, J. Perry et H. Wettstein, New York: OUP, 1989.

Rossetti, Y., Rode, G. et Boisson, D., "Implicit processing of somaesthetic information: a dissociation between where and how?", in NeuroReport 6, 1995.

Stratton, G. M., "Vision without inversion of the retinal image", in Psychological Review 4, 1897.

Strawson, P., Les individus, trad. franç. A. Shalom et P. Drong, Paris: Editions du Seuil, 1973.

Taylor, C., "The Validity of Transcendental Arguments", in Proceedings of the Aristotelian Society, lxxix, 1978-9.

van Cleve, J. et Frederick, R. (éds), The Philosophy of Right and Left, Dordrecht: Kluwer Academic Publishers, 1991.

Vandeloise, C., L'espace en français, Paris: Seuil, 1986.

Wittgenstein, L., Remarques philosophiques, Paris: Gallimard, 1975. 